\title{
Efficient Low-Order Approximation of First-Passage Time Distributions
}

\author{
David Schnoerr, ${ }^{1}$ Botond Cseke, ${ }^{3}$ Ramon Grima, ${ }^{2}$ and Guido Sanguinetti ${ }^{1, *}$ \\ ${ }^{1}$ School of Informatics, University of Edinburgh, Edinburgh EH8 9AB, United Kingdom \\ ${ }^{2}$ School of Biological Sciences, University of Edinburgh, Edinburgh EH9 3JH, United Kingdom \\ ${ }^{3}$ Microsoft Research, Cambridge CB1 2FB, United Kingdom
}

(Received 5 June 2017; revised manuscript received 12 September 2017; published 20 November 2017)

\begin{abstract}
We consider the problem of computing first-passage time distributions for reaction processes modeled by master equations. We show that this generally intractable class of problems is equivalent to a sequential Bayesian inference problem for an auxiliary observation process. The solution can be approximated efficiently by solving a closed set of coupled ordinary differential equations (for the low-order moments of the process) whose size scales with the number of species. We apply it to an epidemic model and a trimerization process and show good agreement with stochastic simulations.
\end{abstract}

DOI: 10.1103/PhysRevLett.119.210601

Many systems in nature consist of stochastically interacting agents or particles. Such systems are frequently modeled as reaction processes whose dynamics are described by master equations [1]. There are several examples of the stochastic modeling of reaction processes in the fields of systems biology [2,3], ecology [4], epidemiology [5], social sciences [6], and neuroscience [7]. The mathematical analysis of such stochastic processes, however, is highly nontrivial.

A particularly important quantity of interest is the firstpassage time (FPT), that is, the random time it takes the process to first cross a certain threshold [8,9]. FPT distributions play a crucial role both in the theory of stochastic processes and in their applications across various disciplines, as they allow us to investigate quantitatively the uncertainty in the emergence of system properties within a finite time horizon. For example, the time it takes cells to respond to external signals by expressing certain genes may be modeled as a FPT problem. Different characteristics of this first time distribution, for example, the variance of the FPT, may represent evolutionarily different strategies that organisms adopt to filter fluctuations in the environment [10-12]. Examples from other disciplines include the extinction time of diseases in epidemic models or the time it takes to form a certain number of polymers in polymerization processes.

FPTs for stochastic processes have been of interest in statistical physics for many decades [13]. For certain random walk or spatial diffusion processes, analytic solutions have been derived [14-16]. Recently, analytic results have been found for effective one-dimensional diffusion processes to a target [17-19]. For multidimensional diffusion processes to small targets, approximate solutions

Published by the American Physical Society under the terms of the Creative Commons Attribution 4.0 International license. Further distribution of this work must maintain attribution to the author(s) and the published article's title, journal citation, and DOI. have been derived using singular perturbation methods and matched asymptotic expansions [20-23].

The problem of computing FPT distributions for reaction processes modeled by master equations, however, is much less explored. Generally, no tractable evolution equations exist except for one-variable, one-step processes $[1,13]$ or certain linear and/or catalytic processes [24-26]. For singletime properties of the underlying master equation, efficient approximation methods exist relying on continuous state spaces [27], but it is not clear how to extend them for the computation of FPTs. Spectral methods constitute efficient approximations for small systems [28,29]. Since these methods typically scale with the size of the state space, they are not applicable to large systems. Some existing FPT approaches for master equations consider rare events in single-species systems and/or mean FPTs only [30-33].

In this Letter, we approach the problem of computing FPTs from a novel perspective. We show that the FPT problem can be formulated exactly as a Bayesian inference problem. We achieve this by introducing an auxiliary observation process that determines whether the process has crossed the threshold up to a given time. This novel formulation allows us to derive an efficient approximation scheme that relies on the solution of a small set of ordinary differential equations. We will use this approximation to analyze the FPT distributions in several nontrivial examples. We focus on reaction networks with discrete state spaces modeled by master equations, but the developed method can also be applied to processes with continuous state spaces modeled by Fokker-Planck equations.

The standard approach to compute the FPT of a process $\boldsymbol{x}_{t}$ to leave a certain region $C$ is to compute the survival probability $Z_{[0, t]}$, that is, the probability that the process remains in $C$ on the time interval $[0, t][13]$. The FPT distribution is then given by the negative time derivative of $Z_{[0, t]}$. The latter can be written as a path integral over the process with an absorbing boundary of $C$ [13]. Equivalently, one can reweigh the unconstrained process by an indicator 
function $p\left(C_{[0, t]} \mid \boldsymbol{x}_{[0, t]}\right)$ on the paths $\boldsymbol{x}_{[0, t]}$ such that $p\left(C_{[0, t]} \mid \boldsymbol{x}_{[0, t]}\right)=1$ if $\boldsymbol{x}_{\tau} \in C$ for $\tau \in[0, t]$ and zero otherwise. One can then write the survival probability $Z_{[0, t]}$ up till time $t$ as a path integral over the density $p\left(\boldsymbol{x}_{[0, t]}\right)$ of the unconstrained process as

$$
Z_{[0, t]}=\int \mathcal{D} \boldsymbol{x}_{[0, t]} p\left(\boldsymbol{x}_{[0, t]}\right) p\left(C_{[0, t]} \mid \boldsymbol{x}_{[0, t]}\right) .
$$

At the heart of our method lies the interpretation of $p\left(C_{[0, t]} \mid \boldsymbol{x}_{[0, t]}\right)$ as a binary observation process: An observer external to the system assesses if the process has left the region of interest or not. In this interpretation, the survival probability $Z_{[0, t]}$ constitutes the marginal likelihood of this auxiliary observation process. The problem of computing $Z_{[0, t]}$ and hence the FPT distribution is thus formally equivalent to a Bayesian inference problem. Note, however, that there are no experimental data involved and no data are being simulated.

Moreover, note that so far no approximations have been made and (1) is exact. However, it is not obvious how to compute the path integral in (1). To make progress, we approximate the continuous-time process with paths $\boldsymbol{x}_{[0, t]}$ by a discrete-time version $\left(\boldsymbol{x}_{t_{0}}, \ldots, \boldsymbol{x}_{t_{N}}\right)$ at points $t_{0}=0, \ldots, t_{N}=$ $t$ with spacing $\Delta t=t / N$. The effects of such a discretization of time on certain survival probabilities has recently been studied in Ref. [34]. We will later take the continuum limit $\Delta t \rightarrow 0$ and are hence not concerned with such effects.

This means that the global observation process $p\left(C_{[0, t]} \mid \boldsymbol{x}_{[0, t]}\right)$ can be written as a product of local observation processes $p\left(C_{t_{i}} \mid \boldsymbol{x}_{t_{i}}\right)$ as

$$
p\left(C_{[0, t]} \mid \boldsymbol{x}_{t_{0}}, \ldots, \boldsymbol{x}_{t_{N}}\right)=\prod_{i=0}^{N} p\left(C_{t_{i}} \mid \boldsymbol{x}_{t_{i}}\right),
$$

where $p\left(C_{t_{i}} \mid \boldsymbol{x}_{t_{i}}\right)=1$ if $\boldsymbol{x}_{t_{i}} \in C$ and zero otherwise. This gives the model a Markovian structure and allows us to cast it into a sequential Bayesian inference problem, as follows. First, we approximate the binary observation factors in (2) by a smooth approximation of the form

$$
p\left(C_{t_{i}} \mid \boldsymbol{x}_{t_{i}}\right) \approx \exp \left[-\Delta t U\left(\boldsymbol{x}_{t_{i}}, t_{i}\right)\right],
$$

where $U\left(\boldsymbol{x}_{t_{i}}, t_{i}\right)$ is a smooth function that is large for $\boldsymbol{x}_{t_{i}} \notin C$ and close to zero for $\boldsymbol{x}_{t_{i}} \in C$, with a sharp slope at the boundary. Moreover, we require $U\left(\boldsymbol{x}_{t_{i}}, t_{i}\right)$ to have a tractable expectation with respect to a normal distribution. The smooth approximation in (3) proves computationally expedient in the algorithm below and will allow us to take the continuum limit $\Delta t \rightarrow 0$. Note that this approximation is equivalent to approximating the global binary constraint with the global soft (that is, continuous) constraint

$$
p\left(C_{[0, t]} \mid \boldsymbol{x}_{[0, t]}\right)=\exp \left(-\int_{0}^{t} d \tau U\left(\boldsymbol{x}_{\tau}, \tau\right)\right) .
$$

The survival probability $Z_{[0, t]}$ in (1) for the discrete-time system factorizes as

$$
Z_{[0, t]} \approx p\left(C_{t_{0}}\right) \prod_{i=0}^{N-1} p\left(C_{t_{i+1}} \mid C_{\leq t_{i}}\right),
$$

where $p\left(C_{t_{0}}\right)$ is the probability of being in $C$ at time $t_{0}$ and $p\left(C_{t_{i+1}} \mid C_{\leq t_{i}}\right) \equiv p\left(C_{t_{i+1}} \mid C_{t_{i}}, C_{t_{i-1}}, \ldots, C_{t_{0}}\right)=\int d \boldsymbol{x}_{t_{i+1}} p\left(C_{t_{i+1}} \mid\right.$ $\left.\boldsymbol{x}_{t_{i+1}}\right) p\left(\boldsymbol{x}_{t_{i+1}} \mid C_{\leq t_{i}}\right)$ is the probability that the process is found to be in $C$ at time $t_{i+1}$, given that it was in $C$ for all previous time points. The computation of these factors corresponds to a sequential Bayesian inference problem which can be solved by iteratively (i) solving the master equation forward between measurement points and (ii) updating the distribution using the observation model. More specifically, the two steps comprise (i) Suppose we know $p\left(\boldsymbol{x}_{t_{i}} \mid C_{\leq t_{i}}\right) \equiv$ $p\left(\boldsymbol{x}_{t_{i}} \mid C_{t_{i}}, C_{t_{i-1}}, \ldots, C_{t_{0}}\right)$ at time $t_{i}$, that is, the marginal distribution of the process at time $t_{i}$ conditioned on the current and all previous observations. Suppose further that, using this as the initial distribution, we can solve the system (the master equation) forward in time until time point $t_{i+1}$ to obtain $p\left(\boldsymbol{x}_{t_{i+1}} \mid C_{\leq t_{i}}\right)$, that is, the marginal distribution of the process at time $t_{i+1}$ conditioned on previous observations [note that $p\left(\boldsymbol{x}_{t_{i+1}} \mid C_{\leq t_{i}}\right)$ does not include the observation $C_{t_{i+1}}$ at time $\left.t_{i+1}\right]$. (ii) To obtain $p\left(\boldsymbol{x}_{t_{i+1}} \mid C_{\leq t_{i+1}}\right)$, we need to take the observation $p\left(C_{t_{i+1}} \mid \boldsymbol{x}_{t_{i+1}}\right)$ at time point $t_{i+1}$ into account. This is achieved by means of Bayes' theorem as

$$
p\left(\boldsymbol{x}_{t_{i+1}} \mid C_{\leq t_{i+1}}\right)=\frac{p\left(C_{t_{i+1}} \mid \boldsymbol{x}_{t_{i+1}}\right) p\left(\boldsymbol{x}_{t_{i+1}} \mid C_{\leq t_{i}}\right)}{Z_{t_{i+1}}}
$$

where we defined the normalization $Z_{t_{i+1}}=p\left(C_{t_{i+1}} \mid C_{\leq t_{i}}\right)$. Note that the latter is just a factor in (5). Performing steps (i) and (ii) iteratively from $t_{0}$ to $t_{N}$ and keeping track of the normalization factors in (6), one can thus, in principle, compute the survival probability according to (5).

However, steps (i) and (ii) are generally intractable, and we propose an approximation method in the following. For step (i), we need to solve the system forward in time. We do this approximately by means of the normal moment closure [35-37], which approximates the discrete process by a continuous one and assumes the single-time probability distribution to be a multivariate normal distribution $\mathcal{N}\left(\boldsymbol{x}_{t} ; \boldsymbol{\mu}_{t}, \boldsymbol{\Sigma}_{t}\right)$ with mean $\boldsymbol{\mu}_{t}$ and covariance $\boldsymbol{\Sigma}_{t}$. Using this assumption on the master equation leads to a closed set of ordinary differential equations for $\boldsymbol{\mu}_{t}$ and $\boldsymbol{\Sigma}_{t}$ which can be solved numerically [27].

Now suppose that we have solved the system forward from time $t$ to $t+\Delta t$ using normal moment closure to obtain $\hat{\boldsymbol{\mu}}_{t+\Delta t}$ and $\hat{\boldsymbol{\Sigma}}_{t+\Delta t}$ and hence the distribution $p\left(\boldsymbol{x}_{t+\Delta t} \mid C_{\leq t}\right)=\mathcal{N}\left(\boldsymbol{x}_{t+\Delta t} ; \hat{\boldsymbol{\mu}}_{t+\Delta t}, \hat{\boldsymbol{\Sigma}}_{t+\Delta t}\right)$ [step (i)]. We next have to perform the observation update in (6) in step (ii) to obtain $p\left(\boldsymbol{x}_{t+\Delta t} \mid C_{\leq t+\Delta t}\right)$. In order to be able to use normal 
moment closure again in the next (i) step, we approximate $p\left(\boldsymbol{x}_{t+\Delta t} \mid C_{\leq t+\Delta t}\right)$ by a multivariate normal distribution with mean $\boldsymbol{\mu}_{t+\Delta t}$ and covariance $\boldsymbol{\Sigma}_{t+\Delta t}$ of the rhs in (6). This approach is known as assumed-density filtering in the statistics literature [38]. In summary, with the described approximations, steps (i) and (ii) comprise (i) Solve normal moment closure equations for $\boldsymbol{\mu}_{t}$ and $\boldsymbol{\Sigma}_{t}$ from time $t$ to $t+\Delta t$ to obtain $\hat{\boldsymbol{\mu}}_{t+\Delta t}$ and $\hat{\boldsymbol{\Sigma}}_{t+\Delta t}$, where $\boldsymbol{\mu}_{t}$ and $\boldsymbol{\Sigma}_{t}$ are, respectively, the mean and covariance of the approximating normal distribution $\mathcal{N}\left(\boldsymbol{x}_{t} ; \boldsymbol{\mu}_{t}, \boldsymbol{\Sigma}_{t}\right)$. (ii) Compute the mean $\boldsymbol{\mu}_{t+\Delta t}$ and covariance $\boldsymbol{\Sigma}_{t+\Delta t}$ of the rhs of (6) and approximate $p\left(\boldsymbol{x}_{t+\Delta t} \mid C_{\leq t+\Delta t}\right)$ in (6) with a corresponding normal distribution $\mathcal{N}\left(\boldsymbol{x}_{t+\Delta t} ; \boldsymbol{\mu}_{t+\Delta t}, \boldsymbol{\Sigma}_{t+\Delta t}\right)$.

Next, we derive a continuous-time description combining steps (i) and (ii). This is achieved by first expanding the update in step (i) leading from $\boldsymbol{\mu}_{t}$ and $\boldsymbol{\Sigma}_{t}$ to $\hat{\boldsymbol{\mu}}_{t+\Delta t}$ and $\hat{\boldsymbol{\Sigma}}_{t+\Delta t}$ in $\Delta t$, which gives a single Euler step update of the moment closure equations. Similarly, we expand step (ii), which leads from $\hat{\boldsymbol{\mu}}_{t+\Delta t}$ and $\hat{\boldsymbol{\Sigma}}_{t+\Delta t}$ to $\boldsymbol{\mu}_{t+\Delta t}$ and $\boldsymbol{\Sigma}_{t+\Delta t}$, as follows. Note first that, by definition, the normalization $Z_{t+\Delta t}$ in (6) can be written as

$$
Z_{t+\Delta t} \approx \int d \boldsymbol{x} \mathcal{N}\left(\boldsymbol{x} ; \hat{\boldsymbol{\mu}}_{t+\Delta t}, \hat{\boldsymbol{\Sigma}}_{t+\Delta t}\right) e^{-\Delta t U(\boldsymbol{x}, t+\Delta t)} .
$$

Taking the logarithm of both sides, expanding in $\Delta t$, and taking derivatives with respect to $\hat{\boldsymbol{\mu}}_{t}$ and $\hat{\boldsymbol{\Sigma}}_{t}$, one can derive the desired expansion of the update in step (ii). The resulting expansions of steps (i) and (ii) can then be combined to give unifying update equations for $\boldsymbol{\mu}_{t}$ and $\boldsymbol{\Sigma}_{t}$ (see Supplemental Material [39] for a derivation). Finally, taking the continuum limit $\Delta t \rightarrow 0$ gives the following closed set of differential equations:

$$
\begin{gathered}
\frac{\partial}{\partial t} \boldsymbol{\mu}_{t}=\left(\frac{\partial}{\partial t} \boldsymbol{\mu}_{t}\right)^{\mathrm{MC}}-\boldsymbol{\Sigma}_{t} \frac{\partial}{\partial \boldsymbol{\mu}_{t}}\left\langle U\left(\boldsymbol{x}_{t}, t\right)\right\rangle_{\mathcal{N}\left(\boldsymbol{x}_{t} ; \boldsymbol{\mu}_{t}, \boldsymbol{\Sigma}_{t}\right)} \\
\frac{\partial}{\partial t} \boldsymbol{\Sigma}_{t}=\left(\frac{\partial}{\partial t} \boldsymbol{\Sigma}_{t}\right)^{\mathrm{MC}}-2 \boldsymbol{\Sigma}_{t}\left(\frac{\partial}{\partial \boldsymbol{\Sigma}_{t}}\left\langle U\left(\boldsymbol{x}_{t}, t\right)\right\rangle_{\mathcal{N}\left(\boldsymbol{x}_{t} ; \boldsymbol{\mu}_{t}, \boldsymbol{\Sigma}_{t}\right)}\right) \boldsymbol{\Sigma}_{t} \\
\frac{\partial}{\partial t} \log Z_{[0, t]}=-\left\langle U\left(\boldsymbol{x}_{t}, t\right)\right\rangle_{\mathcal{N}\left(\boldsymbol{x}_{t} ; \boldsymbol{\mu}_{t}, \boldsymbol{\Sigma}_{t}\right)}
\end{gathered}
$$

Here, the first terms on the rhs of (8) and (9) are, respectively, the equations for the mean and covariance as obtained from the normal moment closure (MC) for the unconstrained system, while the second terms incorporate the auxiliary observation. Equation (10) gives the desired survival probability for the process. We term this method for computing FPT distributions Bayesian first-passage times (BFPT).

Equations (8)-(10) are the central result of this Letter. They constitute closed form ordinary differential equations for the mean, covariance, and log-survival probability of the process, for which efficient numerical integrators exist. Solving these equations forward in time on an interval
$[0, t]$ provides an approximation of the survival probability $Z_{[0, \tau]}$ for all $\tau \in[0, t]$ (on the time grid of the numerical integrator), from which the FPT distribution $p(\tau ; C)$ can be derived for all $\tau \in[0, t]$ by taking the negative derivative of $Z_{[0, \tau]}$, that is, $p(\tau ; C)=-\partial Z_{[0, \tau]} / \partial \tau$. The number of equations scales with the square of the number of species, and the method hence is applicable to large systems. Crucially, and in contrast to stochastic simulations and spectral methods, the complexity of the method is independent of the population size and the size of the state space. Similar equations were obtained in a different context in Refs. [40,41] by means of a variational approximation.

In the derivation of (8)-(10), we utilized three approximations: After discretizing time, we approximated the unconstrained process using normal moment closure and the observation updates by projections onto a normal distribution. We then approximated the binary observation model by a soft loss function, which allowed us to take the continuum limit in time. Depending on the problem, the relative contribution of the three sources to the overall error may vary.

The choice of loss function $U(\boldsymbol{x}, t)$ depends on the problem at hand. In general, for computational convenience one needs to be able to compute analytically the expectation of the loss function with respect to a multivariate normal distribution. In our examples, we use an exponential loss function to constrain the $i$ th component of the state vector $\boldsymbol{x}$ about a threshold $c$ :

$U(\boldsymbol{x}, t)=\exp \left[a\left(x^{i}-c\right)\right], \quad a \in \mathbb{R}, \quad c \in \mathbb{R}$.

The absolute value of the parameter $a$ determines the steepness of the loss function. In principle, we choose $a$ as large as numerically feasible. For a detailed discussion on the choice of the loss function, see Supplemental Material [39].

We now examine the performance of BFPT on three examples. For the analytically tractable Poisson birth process, we find that BFPT captures the low-order moments and the bulk mass of the distribution accurately while giving the correct scaling law for the tail of the distribution (see Supplemental Material [39] for details).

Next, we consider an epidemic system consisting of a susceptible population $S$, an infected population $I$, and a recovered population $R$ and interactions

$$
S+I \stackrel{k_{1}}{\rightarrow} 2 I, \quad I \stackrel{k_{2}}{\rightarrow} R .
$$

This system is frequently modeled as a continuous-time Markov-jump process to model a disease spreading through a population. $k_{1}$ and $k_{2}$ in (12) are the corresponding rate constants. Let $\boldsymbol{x}_{t}=\left(x_{t}, y_{t}, z_{t}\right)$, where $x_{t}, y_{t}$, and $z_{t}$ denote the populations of $S, I$, and $R$, respectively. We are interested in the probability distribution of time for the disease to be permanently eradicated, that is, the time it takes the process to reach a state with $y_{t}=0$. 
Figure 1(b) shows the mean, variance, and mode of the FPT to extinction as obtained from our method and the stochastic simulation algorithm [42]. We find that BFPT accurately captures the mean, variance, and mode of the FPT over a wide range of varying initial values for $S$ and $I$.

Figures 1(c) and 1(d) show the FPT distributions for four different parameter sets. The modality, mode, and overall shape of the FPT are well captured, even for highly skewed and bimodal distributions [cf. the blue curves in Figs. 1(c) and 1(d), respectively]. In some cases, the method predicts less peaked distributions than actual (not shown here). Figures 1(e) and 1(f) show the same results on the logarithmic scale. We observe that our method correctly predicts an exponential scaling (straight lines in the logarithmic scale), although the scaling is not always accurate, indicating a worse approximation in the tails of the distribution.

The value of the approach is borne out by considering its computational efficiency: For the results shown in Fig. 1, BFPT is several orders of magnitude faster than stochastic simulations. For example, simulating $10^{7}$ paths to obtain the results shown in Figs. 1(c)-1(f) takes about $10^{3}-10^{4} \mathrm{~s}$ (a)
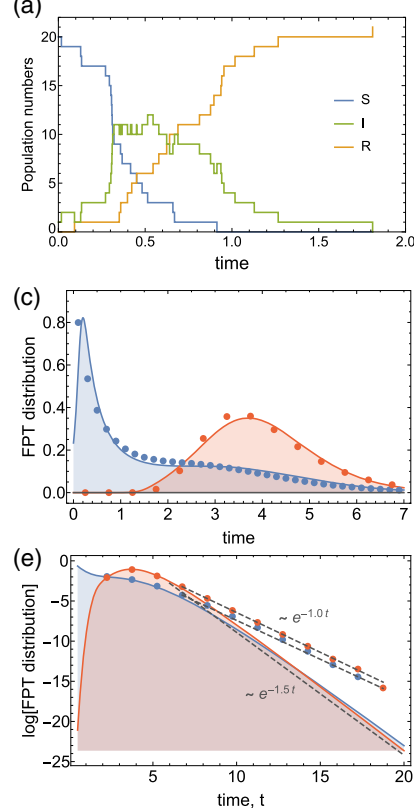
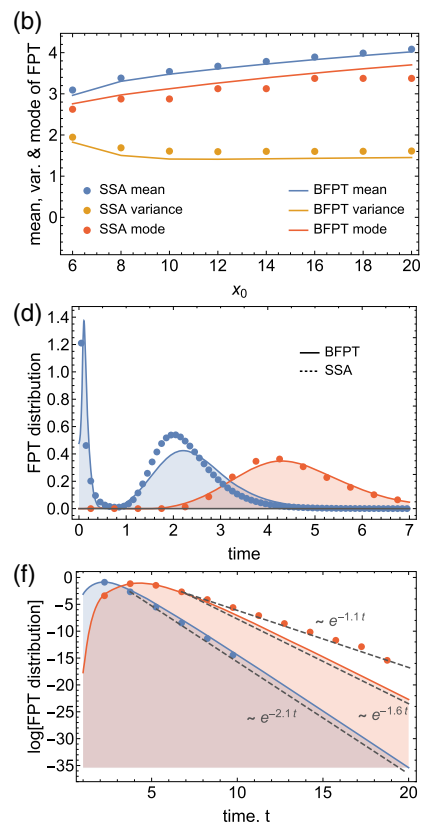

FIG. 1. Results for the epidemic system (12). (a) Simulated path of the process. (b) Mean, variance, and mode of the FPT distribution of species $I$ becoming extinct as a function of the initial populations $x_{0}$ of species $S$, from the stochastic simulation algorithm (SSA, dots, $10^{6}$ samples per point) and from BFPT (lines). The rate constants are set to $k_{1}=0.5$ and $k_{2}=1$, and the initial value for species $I$ is set to $y_{0}=2 x_{0}$. (c),(d) FPT distributions as obtained from the SSA (dots, $10^{7}$ samples per parameter set) and BFPT (lines) for the parameter set $\left(x_{0}, y_{0}, k_{1}, k_{2}\right)$ chosen as $(6,1,0.25,1)$ [blue, (c)], $(20,10,0.5,1)$ [red, (c)], $(20,1,0.5,2)$ [blue, (d)], and $(40,10,0.25,1)$ [red, (d)]. The parameter $a$ in (11) was chosen as $a=-3$ for the blue curve in (c) and $a=-1.5$ for all other figures. (e),(f) The same results as (c),(d) but logarithmic scale. in our implementation of the direct stochastic simulation algorithm [42], whereas BFPT takes less than a second.

Finally, we apply BFPT to a polymerization system of monomers $X$, dimers $X X$, and trimers $X X X$ with interactions

$$
X+X \stackrel{k_{1}}{\rightarrow} X X, \quad X X+X \stackrel{k_{2}}{\rightarrow} X X X .
$$

Starting from a fixed number of $10^{3}$ of monomers, zero dimers, and zero trimers, we are interested in the FPT it takes to produce 200 trimers. We are interested in exploring the dependence of this FPT distribution on the parameters of the system (dimerization and trimerization rate $k_{1}$ and $k_{2}$, respectively); such parameter exploration is computationally too demanding to be performed by a brute force simulation without access to dedicated hardware, since the FPT distribution needs to be estimated for a large number of parameter sets.

Figure 2 shows the results for this process. We observe excellent agreement between BFPT and simulations for a particular value of the parameters [Fig. 2(a)]. The heat plot for the mean as a function of $k_{1}$ and $k_{2}$ indicates that for a given trimerization rate $k_{2}$ a minimal mean FPT is achieved for an intermediate value of dimerization rate $k_{1}$ [Fig. 2(b)]. We find a linear relationship $k_{2} \approx 2.3 k_{1}$ between the two
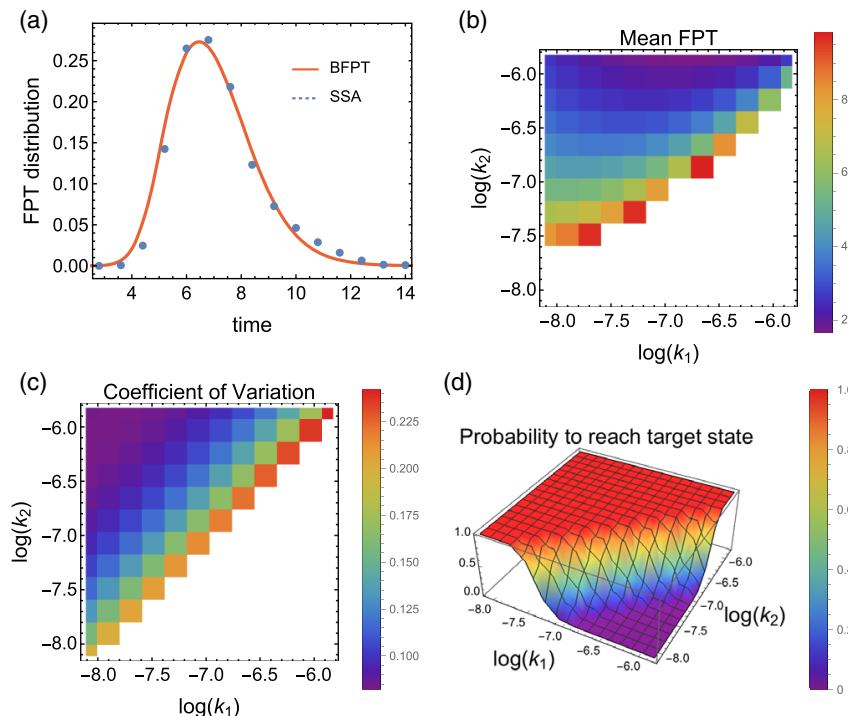

(d)

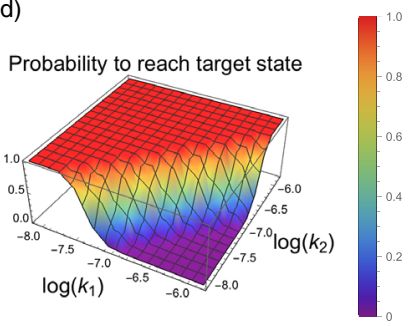

FIG. 2. Results for the polymerization system in (13). (a) FPT distribution for the parameters $k_{1}=k_{2}=10^{-3}$ obtained from the SSA (dots, $10^{4}$ samples). (b),(c) Heat plots of the mean and the coefficient of variation (defined as the standard deviation divided by the mean) of the FPT to produce 200 trimers starting with $10^{3}$ monomers, as a function of $k_{1}$ and $k_{2}$ on the logarithmic scale. (d) Corresponding 3D plot for the normalization of the FPT distribution, that is, the probability with which at least 200 trimers are being produced. The white areas in (b) and (c) indicate that either the value is larger than the plotted range or that the target state is reached with such small probability that an estimation of moments is not sensible. The parameter $a$ in (11) was fixed to $a=0.2$ for all figures. 
rates for the location of these minima. The variance of FPT behaves quantitatively similarly (not shown in the figure). The coefficient of variation [Fig. 2(c)], however, becomes minimal for small values of $k_{1}$ for a given $k_{2}$. This reveals an unexpected trade-off between an optimal mean FPT and optimal noise-to-mean ratio (coefficient of variation). Figure 2(d) shows the probability that the target state is reached, that is, the probability that at least 200 trimers are being produced. We find that there are two parameter regions, one with a probability close to one and one with a probability close to zero, and a small transition range between these two with boundary $k_{2} \approx 0.55 k_{1}$.

In conclusion, we have shown that the problem of computing survival probabilities and FPT distributions for stochastic processes can be formulated as a sequential Bayesian inference problem. This novel formulation opens the way for a new class of efficient approximation methods from machine learning and computational statistics to address this classical intractable problem. Here, we derived an approximation for FPT distributions which relies on solving a small set of ordinary differential equations. This results in considerable efficiency gains; empirically, we found the approximation to be highly accurate in several examples. However, we do not have at present systematic error estimates for the method; we leave the investigation of such bounds and possible correction methods for future work. In particular, it will be interesting to study the tail behavior of FPT distributions with our method, as these were not always captured well in our examples. We notice that, while we applied our method to processes with discrete state spaces modeled by master equations, in principle, it can equally easily be applied to processes with continuous state spaces modeled by Fokker-Planck equations.

This work was supported by the Leverhulme Trust [RPG-2013-171] and the European Research Council [MLCS 306999]. We thank Manfred Opper for insightful discussions.

*gsanguin@inf.ed.ac.uk

[1] C. W. Gardiner, Handbook of Stochastic Methods (Springer, Berlin, 1985), Vol. 3.

[2] A. Eldar and M. B. Elowitz, Functional roles for noise in genetic circuits, Nature (London) 467, 167 (2010).

[3] R. Grima, Noise-Induced Breakdown of the MichaelisMenten Equation in Steady-State Conditions, Phys. Rev. Lett. 102, 218103 (2009).

[4] A. J. McKane and T. J. Newman, Predator-Prey Cycles from Resonant Amplification of Demographic Stochasticity, Phys. Rev. Lett. 94, 218102 (2005).

[5] M. I. Dykman, I. B. Schwartz, and A. S. Landsman, Disease Extinction in the Presence of Random Vaccination, Phys. Rev. Lett. 101, 078101 (2008).

[6] J. Fernández-Gracia, K. Suchecki, J. J. Ramasco, M. San Miguel, and V. M. Eguíluz, Is the Voter Model a Model for Voters?, Phys. Rev. Lett. 112, 158701 (2014).
[7] T. Betz, D. Lim, and J. A. Käs, Neuronal Growth: A Bistable Stochastic Process, Phys. Rev. Lett. 96, 098103 (2006).

[8] M. R. D'Orsogna and T. Chou, First Passage and Cooperativity of Queuing Kinetics, Phys. Rev. Lett. 95, 170603 (2005).

[9] S. Condamin, O. Bénichou, V. Tejedor, R. Voituriez, and J. Klafter, First-passage times in complex scale-invariant media, Nature (London) 450, 77 (2007).

[10] E. Kussell and S. Leibler, Phenotypic diversity, population growth, and information in fluctuating environments, Science 309, 2075 (2005).

[11] S. Tănase-Nicola, P. B. Warren, and P. R. ten Wolde, Signal Detection, Modularity, and the Correlation between Extrinsic and Intrinsic Noise in Biochemical Networks, Phys. Rev. Lett. 97, 068102 (2006).

[12] T. J. Kobayashi, Implementation of Dynamic Bayesian Decision Making by Intracellular Kinetics, Phys. Rev. Lett. 104, 228104 (2010).

[13] S. Redner, A Guide to First-Passage Processes (Cambridge University Press, Cambridge, England, 2001).

[14] O. Bénichou, C. Loverdo, M. Moreau, and R. Voituriez, Intermittent search strategies, Rev. Mod. Phys. 83, 81 (2011).

[15] A. J. Bray, S. N. Majumdar, and G. Schehr, Persistence and first-passage properties in nonequilibrium systems, Adv. Phys. 62, 225 (2013).

[16] F. Aurzada and T. Simon, Persistence probabilities and exponents, in Lévy Matters V (Springer, New York, 2015), pp. 183-224.

[17] A. Godec and R. Metzler, Universal Proximity Effect in Target Search Kinetics in the Few-Encounter Limit, Phys. Rev. X 6, 041037 (2016).

[18] A. Godec and R. Metzler, First passage time distribution in heterogeneity controlled kinetics: going beyond the mean first passage time, Sci. Rep. 6, 20349 (2016).

[19] A. Godec and R. Metzler, First passage time statistics for two-channel diffusion, J. Phys. A 50, 084001 (2017).

[20] A. Singer, Z. Schuss, D. Holcman, and R. Eisenberg, Narrow escape, part I, J. Stat. Phys. 122, 437 (2006).

[21] D. Holcman and Z. Schuss, The narrow escape problem, SIAM Rev. 56, 213 (2014).

[22] S. A. Isaacson, A. J. Mauro, and J. Newby, Uniform asymptotic approximation of diffusion to a small target: Generalized reaction models, Phys. Rev. E 94, 042414 (2016).

[23] P. C. Bressloff and S. D. Lawley, Residence times of a Brownian particle with temporal heterogeneity, J. Phys. A 50, 195001 (2017).

[24] G. Bel, B. Munsky, and I. Nemenman, The simplicity of completion time distributions for common complex biochemical processes, Phys. Biol. 7, 016003 (2009).

[25] B. Munsky, I. Nemenman, and G. Bel, Specificity and completion time distributions of biochemical processes, J. Chem. Phys. 131, 235103 (2009).

[26] R. Grima and A. Leier, Exact product formation rates for stochastic enzyme kinetics, J. Phys. Chem. B 121, 13 (2017).

[27] D. Schnoerr, G. Sanguinetti, and R. Grima, Approximation and inference methods for stochastic biochemical kinetics-a tutorial review, J. Phys. A 50, 093001 (2017).

[28] P. Deuflhard, W. Huisinga, T. Jahnke, and M. Wulkow, Adaptive discrete galerkin methods applied to the chemical master equation, SIAM J. Sci. Comput. 30, 2990 (2008). 
[29] V. Kazeev, M. Khammash, M. Nip, and C. Schwab, Direct solution of the chemical master equation using quantized tensor trains, PLoS Comput. Biol. 10, e1003359 (2014).

[30] M. Assaf, A. Kamenev, and B. Meerson, Population extinction in a time-modulated environment, Phys. Rev. E 78, 041123 (2008).

[31] M. Assaf and B. Meerson, Extinction of metastable stochastic populations, Phys. Rev. E 81, 021116 (2010).

[32] S. Be'er and M. Assaf, Rare events in stochastic populations under bursty reproduction, J. Stat. Mech. (2016) 113501.

[33] M. F. Weber and E. Frey, Master equations and the theory of stochastic path integrals, Rep. Prog. Phys. 80, 046601 (2017).

[34] S. N. Majumdar, A. J. Bray, and G. C. M. A. Ehrhardt, Persistence of a continuous stochastic process with discrete-time sampling, Phys. Rev. E 64, 015101 (2001).

[35] L. A. Goodman, Population growth of the sexes, Biometrics 9, 212 (1953).

[36] D. Schnoerr, G. Sanguinetti, and R. Grima, Validity conditions for moment closure approximations in stochastic chemical kinetics, J. Chem. Phys. 141, 084103 (2014).
[37] D. Schnoerr, G. Sanguinetti, and R. Grima, Comparison of different moment-closure approximations for stochastic chemical kinetics, J. Chem. Phys. 143, 185101 (2015).

[38] P. S. Maybeck, Stochastic Models, Estimation, and Control (Academic, New York, 1982), Vol. 3.

[39] See Supplemental Material at http://link.aps.org/ supplemental/10.1103/PhysRevLett.119.210601 for a derivation of (8)-(10) and a detailed discussion of the choice for the loss function in (11), as well as a derivation of the exact results for the Poisson birth process.

[40] B. Cseke, M. Opper, and G. Sanguinetti, Approximate inference in latent Gaussian-Markov models from continuous time observations, in Advances in Neural Information Processing Systems (Nips Foundation, Lake Tahoe, 2013), pp. 971-979.

[41] B. Cseke, D. Schnoerr, M. Opper, and G. Sanguinetti, Expectation propagation for continuous time stochastic processes, J. Phys. A 49, 494002 (2016).

[42] D. T. Gillespie, A general method for numerically simulating the stochastic time evolution of coupled chemical reactions, J. Comput. Phys. 22, 403 (1976). 